\title{
Prognostic Factors in COVID-19 Pneumonia with Severe Acute Respiratory Distress Syndrome: An Observational Study
}

\author{
Xiaowen Wang ${ }^{*}$, Liya Zhu ${ }^{2 *}$, Jian Liu ${ }^{1}$, Keming Chen ${ }^{1}$, Xiangqiong Liu ${ }^{3}$,

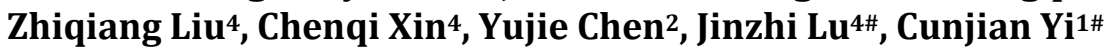

${ }^{1}$ Department of Obstetrics and Gynecology, The First Affiliated Hospital of Yangtze University, Jingzhou, China ${ }^{2}$ Department of Endocrinology, The First Affiliated Hospital of Yangtze University, Jingzhou, China ${ }^{3}$ Department of Laser Ophthalmology, The First Affiliated Hospital of Yangtze University, Jingzhou, China. ${ }^{4}$ Department of Central Laboratory of Basic Medicine, The First Affiliated Hospital of Yangtze University, Jingzhou, China Email: "cunjiany@163.com, ${ }^{\sharp}$ Jinzhilu2015@163.com

How to cite this paper: Wang, X.W., Zhu, L.Y., Liu, J., Chen, K.M., Liu, X.Q., Liu, Z.Q., Xin, C.Q., Chen, Y.J., Lu, J.Z. and Yi, C.J. (2021) Prognostic Factors in COVID-19 Pneumonia with Severe Acute Respiratory Distress Syndrome: An Observational Study. Yangtze Medicine, 5, 249-265.

https://doi.org/10.4236/ym.2021.54024

Received: June 4, 2021

Accepted: December 24, 2021

Published: December 27, 2021

Copyright $\odot 2021$ by author(s) and Scientific Research Publishing Inc. This work is licensed under the Creative Commons Attribution International License (CC BY 4.0).

http://creativecommons.org/licenses/by/4.0/

\begin{abstract}
Purpose: To identify prognostic factors in patients with coronavirus disease 2019 (COVID-19)-associated severe acute respiratory distress syndrome (ARDS). Methods: In this study, we included 45 patients with COVID-19associated ARDS hospitalized at The First Affiliated Hospital of Yangtze University in Jingzhou, Hubei, China, between January 22, 2020, and March 6, 2020. Clinical data and outcomes were reviewed and analyzed according to the Berlin definition. Findings: Men were more likely to develop severe ARDS than women $(11[91.7 \%]$ vs. 1 [8.3\%]). Factors associated with severe ARDS included sex (male) (hazard ratio [HR], 13.75; 95\% confidence interval [CI], 1.45 - 130.24), neutrophil count (HR, 55.00; 95\% CI, 5.02 - 602.15), lymphocyte count (HR, 40.00; 95\% CI, 4.83 - 331.00), prothrombin time (HR, 12.14; 95\% CI, 1.19 - 123.62), D-dimer (HR, 11.00; 95\% CI, 1.16 - 103.94), total bilirubin levels (HR, 5.00; 95\% CI, 0.93 - 26.79), albumin (HR, 17.5; 95\% CI, 2.67 - 114.85), blood urea nitrogen levels (HR, 28.60; 95\% CI, 2.89 283.06), lactate dehydrogenase levels (HR, 6.00; 95\% CI, 1.17 - 30.73), and C-reactive protein levels (HR, 15.87; 95\% CI, 2.40 - 111.11). Conclusion: Laboratory indicators, such as neutrophil count and lymphocyte count, could play an important role in the diagnosis of severe ARDS and guide clinical decision-making for patients with ARDS.
\end{abstract}

\section{Keywords}

Acute Respiratory Distress Syndrome, Coronavirus Disease 2019, Pneumonia, Prognostic Factors

\footnotetext{
${ }^{*}$ Xiaowen Wang, Liya Zhu and Jian Liu are joint first authors.

${ }^{\#}$ Corresponding author.
} 


\section{Introduction}

Coronavirus disease 2019 (COVID-19) is a recently emerged infectious disease caused by a novel coronavirus $(2019-\mathrm{nCoV})$. The disease has spread rapidly throughout Wuhan (Hubei province) and the world [1] [2] [3]. Several studies reported that patients with severe COVID-19 are more likely to develop acute respiratory distress syndrome (ARDS) and have higher mortality [4] [5] [6]. For example, among 52 critically ill adult patients with COVID-19 in Wuhan Jin Yin-tan hospital (Wuhan, China), 35 patients (67\%) developed ARDS, 26 of whom (81\%) died [4]. Similarly, among 36 COVID-19 patients hospitalized in the intensive care unit of Zhongnan Hospital of Wuhan University, 22 (61.1\%) developed ARDS, 6 of whom (27.2\%) eventually died [5].

ARDS results from direct (e.g., pneumonia or aspiration) or indirect (e.g., extrapulmonary sepsis) lung injury and has a high mortality rate [7]. Clinically, patients with ARDS develop severe hypoxemia and hypercapnia, and most die of sepsis or multiorgan failure rather than refractory respiratory failure. Hospital mortality of $40 \%$ has been reported in patients with ARDS [7] [8]. According to the Berlin definition, ARDS is classified into three severity levels (i.e., mild, moderate, and severe) based on the degree of hypoxemia [9]. However, there are no prognostic factors in COVID-19-associated severe ARDS. In this study, we evaluated patients with ARDS due to COVID-19 who were admitted to The First Affiliated Hospital of Yangtze University in Jingzhou, Hubei, China. The objective of this case series was to identify prognostic factors in patients with COVID19-associated severe ARDS according to the Berlin definition [9]. The baseline ARDS morbidity and mortality reported in this study will be of considerable value for the early identification of individuals who are at risk of developing severe ARDS and who are most likely to benefit from further treatment.

\section{Methods}

\subsection{Study Design and Participants}

This single-center, retrospective, observational study was conducted at The First Affiliated Hospital of Yangtze University (Jingzhou, Hubei Province), a designated center for the treatment of patients with COVID-19. We retrospectively analyzed data from 45 patients with COVID-19 who developed ARDS between January 23 and March 6, 2020. Laboratory confirmation of COVID-19 was performed at The First Affiliated Hospital of Yangtze University according to WHO interim guidelines [10]. ARDS diagnosis was performed according to the Berlin definitions [9]. Patients who died within $24 \mathrm{~h}$ of ARDS diagnosis, children, and adolescents were excluded. Our analyses included 15 patients with mild ARDS, 18 with moderate ARDS, and 12 with severe ARDS. All chest CT images were reviewed by experienced radiologists. Based on the degree of hypoxemia, ARDS severity was determined as mild $(200 \mathrm{mmHg}<\mathrm{PF}$ ratio $\leq 300 \mathrm{mmHg})$, moderate $(100 \mathrm{mmHg}<\mathrm{PF}$ ratio $\leq 200 \mathrm{mmHg}$ ), or severe (PF ratio $<100 \mathrm{mmHg}$ ). This retrospective study was approved by the Ethics Committee of the First Affiliated 
Hospital of Yangtze University (No. K20200102). Verbal consent was obtained from all patients.

\subsection{Data Collection}

We reviewed the electronic medical records of all patients with laboratory-confirmed COVID-19. Recorded data included demographic information, medical history, exposure history, comorbidities, symptoms, signs, laboratory findings, treatments, and outcomes. The majority of clinical data regarding systolic pressure, respiratory rate, blood routine blood test, coagulation, biochemical tests, chest CT scans, and partial pressure of arterial oxygen $\left(\mathrm{PaO}_{2}\right) /$ fraction of inspired oxygen $\left(\mathrm{FIO}_{2}\right)$ were obtained within 24 hours of ARDS diagnosis. Shock was defined according to the guidelines of WHO for novel coronavirus disease 2019 (COVID-19) [4] [10], while acute kidney injury was diagnosed based on serum creatinine levels (KDIGO clinical practice guidelines for acute kidney injury, 2012) [11]. Cardiac injury was diagnosed if the serum concentration of hypersensitive cardiac troponin I (hsTNI) was above the upper limit of the reference range $(34.2 \mathrm{pg} / \mathrm{mL})$ [4]. All clinical data were reviewed by an experienced team of physicians. Any missing or uncertain records were collected and clarified through direct communication with involved healthcare providers and the families of the patients.

\subsection{Statistical Analysis}

Continuous variables were expressed as medians and interquartile range (IQR), and comparisons were performed using the Kruskal-Wallis $\mathrm{H}$ test. Categorical variables were expressed as numbers (\%) and compared using the chi-squared test or Fisher's exact test among patients with mild, moderate, and severe ARDS. Kaplan Meier plots were created for survival data, and significance in survival data was determined using the Mantel-Cox (log-rank) test. Multivariate Cox proportional hazard ratio (HR) models were used to identify factors associated with ARDS development. All statistical tests were two-sided, with the significance threshold set at $\alpha<0.05$. Statistical analyses were conducted using the SPSS software, version 17.0.

\section{Results}

\subsection{Clinical Characteristics of Patients}

In this study, we retrospectively analyzed data from 45 COVID-19 patients who developed ARDS between January 23 and March 6, 2020; no children or adolescents were included in the study. All study participants were residents of Jing Zhou City. The median age was 64 years (IQR, 53 - 73; range, 18 - 88 years); 63 (IQR, 54 - 72) for patients with mild ARDS, 65 (IQR, 47 - 75) for patients with moderate, and 66 (IQR, 52 - 73) for patients with severe ARDS. The ages of the three ARDS groups did not differ significantly $(\mathrm{p}=0.929)$. Twenty-two $(48.9 \%)$ patients were older than 65 years (6 [40\%] had mild ARDS, 9 [50\%] had mod- 
erate ARDS, and 7 [58.3\%] had severe ARDS). Twenty-three (51.1\%) patients were younger than 65 years (9 [60\%] had mild ARDS, 9 [50\%] had moderate ARDS, and 5 [41.7\%] had severe ARDS). Severe ARDS was not significantly associated with age $(\mathrm{p}=0.644)$. Twenty-nine $(64.4 \%)$ patients were men (mild: 7 [46.7\%], moderate: 11 [61.1\%], severe: 11 [91.7\%]), and 16 (35.6\%) patients were women (mild: 8 [53.3\%], moderate: 7 [38.9\%], severe: 1 [8.3\%]). Compared with women, men were more likely to develop severe ARDS (11 [91.7\%] in men vs. 1 [8.3\%] in women, $\mathrm{p}=0.044)$. Thirty $(66.7 \%)$ patients had a contact history with the epidemic area of Wuhan (mild: 8 [53.3\%], moderate: 12 [66.7\%], severe: 10 [83.3\%]). Fifteen (33.3\%) patients had never left from the epidemic area of Jingzhou (mild: 7 [46.7\%], moderate: 6 [33.3\%], severe: 2 [16.7\%]). The median duration between the first symptoms and ARDS development was 8 days (IQR, 3 - 16) (mild: 8 [IQR, 2 - 19], moderate: 7 [IQR, 4 - 16], severe: 7 [IQR, 2 - 15]; Table 1).

Thirty of 45 (66.7\%) patients with ARDS had at least one chronic disease, including diabetes $(\mathrm{n}=4,8.9 \%$; mild: 1 [6.7\%], moderate: 2 [11.1\%], severe: 1 [8.3\%]), hypertension (17, 37.8\%; mild 6 [40.0\%], moderate: 7 [38.9\%], severe: 4 [33.4\%]), cardiovascular disease $(4,8.9 \%$; mild: 1 [6.7\%], moderate: 2 [11.1\%], severe: $1[8.3 \%])$, chronic obstructive pulmonary disease $(\mathrm{n}=4,8.9 \%$; mild: 1 [6.7\%], moderate: 3 [16.7\%], severe: $0[0 \%])$, and cancer $(n=1,2.2 \%$; mild: 0 [0\%], moderate: $1[5.6 \%]$, severe: $0[0 \%]$ ), There was no significant association between chronic illness and ARDS severity. Patients with ARDS experienced fever ( $\mathrm{n}=45,100 \%$; mild: 15 [100\%], moderate: 18 [100\%], severe: 12 [100\%]), dry cough ( $\mathrm{n}=41,91.1 \%$; mild: 14 [93.3\%], moderate: 16 [88.9\%], severe: 11 [91.7\%]), dyspnea ( $\mathrm{n}=33,73.3 \%$; mild: 8 [53.3\%], moderate: 13 [72.2\%], severe: 12 [100\%]), vomiting $(\mathrm{n}=20,44.4 \%$; mild: 6 [40\%], moderate: 8 [44.4\%], severe: 6 [50\%]), fatigue ( $\mathrm{n}=22,48.9 \%$; mild: 9 [60\%], moderate: 8 [44.4\%], severe: 5 [41.7\%]), and chest pain $(\mathrm{n}=40,88.9 \%$; mild: 12 [80\%], moderate: 16 [88.9\%], severe: $12[100 \%])$. Less frequent symptoms included hemoptysis $(\mathrm{n}=1,2.2 \%$; mild: $0(0 \%)$, moderate: $0[0 \%]$, severe: $1[8.3 \%])$, dorsalgia $(\mathrm{n}=6,13.3 \%$; mild: 3 [20.0\%], moderate: $1[5.6 \%]$, severe: $2[16.7 \%])$, and diarrhea $(n=8,17.8 \%$; mild: 4 [26.7\%], moderate: 3 [16.7\%], severe: 1 [8.3\%]). Dyspnea was significantly more frequent in patients with severe ARDS than in patients with mild or moderate ARDS (mild: $53.3 \%$, moderate: $72.2 \%$, severe: $100 \%, p=0.017$; Table 1 ).

\subsection{Comorbidities and Prognosis}

The median systolic pressure was $123 \mathrm{mmHg}$ (IQR, 112 - 152) in all patients (mild: $116 \mathrm{mmHg}$ [IQR, 112 - 137], moderate: $124 \mathrm{mmHg}$ [IQR, 113 - 147], severe: $115 \mathrm{mmHg}$ [IQR, 88 - 125]). The median respiratory rate was 30.5 (IQR, 30 - 35; mild: 30 [IQR, 29 - 31], moderate: 34.5 [IQR, 30.3 - 36.5], severe: 33 [IQR, 29.3 - 39]). The median $\mathrm{pH}$ was 7.45 (IQR, 7.42 - 7.48; mild: 7.42 [IQR, 7.4 7.43], moderate: 7.47 [IQR, 7.45 - 7.49], severe: 7.47 [IQR, 7.42 - 7.48]). The median $\mathrm{PO}_{2}$ was $68 \mathrm{mmHg}$ (IQR, 55.5 - 75; mild: 75 [IQR, 72 - 80], moderate: 66.4 
Table 1. Characteristics and symptoms of COVID-19 patients who developed ARDS.

\begin{tabular}{|c|c|c|c|c|c|}
\hline & $\begin{array}{l}\text { All patients } \\
(n=45)\end{array}$ & $\begin{array}{c}\text { Mild } \\
(n=15)\end{array}$ & $\begin{array}{l}\text { Moderate } \\
(\mathrm{n}=18)\end{array}$ & $\begin{array}{l}\text { Severe } \\
(n=12)\end{array}$ & $\mathrm{p}$ values \\
\hline Age, median (IQR), years & $64(53-73)$ & $\begin{array}{c}63(54- \\
72)\end{array}$ & $\begin{array}{c}65(47- \\
75)\end{array}$ & $66(52-73)$ & 0.929 \\
\hline$\leq 65$ & $23(51.1 \%)$ & $9(60.0 \%)$ & $9(50.0 \%)$ & $5(41.7 \%)$ & 0614 \\
\hline$>65$ & $22(48.9 \%)$ & $6(40.0 \%)$ & $9(50.0 \%)$ & $7(58.3 \%)$ & \\
\hline \multicolumn{6}{|l|}{ Sex } \\
\hline Female & $16(35.6 \%)$ & $8(53.3 \%)$ & $7(38.9 \%)$ & $1(8.3 \%)$ & 0.044 \\
\hline Male & $29(64.4 \%)$ & $7(46.7 \%)$ & $11(61.1 \%)$ & $11(91.7 \%)$ & \\
\hline $\begin{array}{l}\text { Onset of symptom to } \\
\text { ARDS, median (IQR), days }\end{array}$ & $8(3-16)$ & $8(2-19)$ & $7(4-16)$ & $7(2-15)$ & 0.944 \\
\hline \multicolumn{6}{|c|}{ Contact history of epidemic area } \\
\hline Wuhan & $30(66.7 \%)$ & $8(53.3 \%)$ & $12(66.7 \%)$ & $10(83.3 \%)$ & 0.238 \\
\hline Jingzhou & $15(33.3 \%)$ & $7(46.7 \%)$ & $6(33.3 \%)$ & $2(16.7 \%)$ & \\
\hline Chronic medical illness & $30(66.7 \%)$ & $9(60.0 \%)$ & $15(83.4 \%)$ & $6(50 \%)$ & \\
\hline Diabetes & $4(8.9 \%)$ & $1(6.7 \%)$ & $2(11.1 \%)$ & $1(8.3 \%)$ & $>0.99$ \\
\hline Hypertension & $17(37.8 \%)$ & $6(40.0 \%)$ & $7(38.9 \%)$ & $4(33.4 \%)$ & $>0.99$ \\
\hline Cardiovascular disease & $4(8.9 \%)$ & $1(6.7 \%)$ & $2(11.1 \%)$ & $1(8.3 \%)$ & $>0.99$ \\
\hline $\begin{array}{l}\text { Chronic obstructive } \\
\text { pulmonary disease }\end{array}$ & $4(8.9 \%)$ & $1(6.7 \%)$ & $3(16.7 \%)$ & $0(0 \%)$ & 0.434 \\
\hline Cancer & $1(2.2 \%)$ & $0(0 \%)$ & $1(5.6 \%)$ & $0(0 \%)$ & $>0.99$ \\
\hline Current smoking & $4(8.9 \%)$ & $1(6.7 \%)$ & $1(5.6 \%)$ & $2(16.7 \%)$ & 0.656 \\
\hline \multicolumn{6}{|l|}{ Signs and symptoms } \\
\hline Fever & $45(100 \%)$ & $15(100 \%)$ & $18(100 \%)$ & $12(100 \%)$ & $>0.99$ \\
\hline Dry Cough & $41(91.1 \%)$ & $14(93.3 \%)$ & $16(88.9 \%)$ & $11(91.7 \%)$ & $>0.99$ \\
\hline Dyspnea & $33(73.3 \%)$ & $8(53.3 \%)$ & $13(72.2 \%)$ & $12(100 \%)$ & 0.017 \\
\hline Vomiting & $20(44.4 \%)$ & $6(40 \%)$ & $8(44.4 \%)$ & $6(50 \%)$ & 0.931 \\
\hline Fatigue & $22(48.9 \%)$ & $9(60 \%)$ & $8(44.4 \%)$ & $5(41.7 \%)$ & 0.6 \\
\hline Chest pain & $40(88.9 \%)$ & $12(80 \%)$ & $16(88.9 \%)$ & $12(100 \%)$ & 0.35 \\
\hline Haemoptysis & $1(2.2 \%)$ & $0(0 \%)$ & $0(0 \%)$ & $1(8.3 \%)$ & 0.272 \\
\hline Dorsalgia & $6(13.3 \%)$ & $3(20.0 \%)$ & $1(5.6 \%)$ & $2(16.7 \%)$ & 0.477 \\
\hline Diarrhea & $8(17.8 \%)$ & $4(26.7 \%)$ & $3(16.7 \%)$ & $1(8.3 \%)$ & 0.486 \\
\hline
\end{tabular}

Data are median (IQR), $\mathrm{n}(\%)$, or $\mathrm{n} / \mathrm{N}(\%)$, where $\mathrm{N}$ is the total number of patients with available data. $P$-values for mild, moderate, and severe groups were calculated using the $\chi^{2}$ test, Fisher's exact test, or Kruskal-Wallis $\mathrm{H}$ test.

[IQR, 59.5 - 74.3], severe: 53.2 [IQR, 45.6 - 56.8]). The median $\mathrm{PCO}_{2}$ was 35.6 mmHg (31.8 - 41.1; mild: 40.2 [IQR, 32.8 - 41.4], moderate: 34.3 [IQR, 31.6 - 
37.2], severe: 35.5 [IQR, 30.4 - 41.2]). The median $\mathrm{PO}_{2} / \mathrm{FiO}_{2}$ was $150 \mathrm{mmHg}$ (IQR, 92.7 - 218; mild: 228 [IQR, 219.5 - 245], moderate: 138.5 [IQR, 106.3 168.4], severe: 78.3 [IQR, 72.5 - 86.8]) (Table 2). Compared with patients with mild and moderate ARDS, patients with severe ARDS had higher median respiratory rate and $\mathrm{pH}(\mathrm{p}=0.039$ for respiratory rate and $\mathrm{p}=0.001$ for $\mathrm{pH})$. Additionally, the median $\mathrm{PO}_{2}$ and $\mathrm{PO}_{2} / \mathrm{FiO}_{2}$ were significantly lower in patients with severe ARDS ( $\mathrm{p}<0.001$ for $\mathrm{PO}_{2}$, and $\mathrm{p}<0.001$ for $\mathrm{PO}_{2} / \mathrm{FiO}_{2}$, respectively) than in patients with mild ARDS. The median systolic pressure and $\mathrm{PCO}_{2}$ did not differ significantly among the three patient groups (Table 2). On the day of ARDS diagnosis, the median Acute Physiology and Chronic Health Evaluation II (APACHE II) was 16 (IQR, 12 - 24; mild: 12 [IQR, 10 - 16], moderate: 16 [IQR, 12 - 20], severe: 28 [IQR, 23 - 37]), the median Glasgow Coma Scale (GCS) was 14 (IQR, 9 - 15; mild: 15 [IQR, 14 - 15], moderate: 12.5 [IQR, 11.25 - 15], severe: 8 [IQR, 7 - 15]), and Sequential Organ Failure Assessment score (SOFA) was 4 (IQR, 3 - 6; mild: 3 [IQR, 2.5 - 4], moderate: 4 [IQR, 3 - 5], severe: 7 [IQR, 5.75 9.25]). Compared with the other two ARDS groups, patients with severe ARDS had significantly higher APACHE II, median GCS, and SOFA ( $p<0.001$ for APACHE II; $p=0.025$ for GCS; $p<0.001$ for SOFA). The SOFA scores of five patients with severe ARDS who died were higher than five (Table 2).

Most COVID-19 patients with ARDS exhibited organ function failure, including acute kidney injury $(\mathrm{n}=7,15.6 \%$; mild: 2 [13.3\%], moderate: 2 [11.1\%], severe: 3 [25\%]), cardiac injury $(n=9,20 \%$; mild: 1 [6.7\%], moderate: 2 [11.1\%], severe: 6 [50\%]), liver dysfunction $(n=20.44 .4 \%$; mild: 4 [26.7\%], moderate: 11 [61.1\%], severe: 5 [41.7\%]), hyperglycemia $(n=12,26.7 \%$; mild: 1 [6.7\%], moderate: 6 [33.3\%], severe: 5 [41.7\%]), pneumothorax $(n=2,4.4 \%$; mild: 0 [0\%], moderate: $0[0 \%]$, severe: $2[16.7 \%])$, shock $(n=5,11.1 \%$; mild: 0 [0\%], moderate: 1 [5.6\%], severe: 4 [33.4\%]; Table 2). Compared with patients with mild and moderate ARDS, patients with severe ARDS had significantly more frequent cardiac injury and shock ( $\mathrm{p}=0.013$ for cardiac injury and $\mathrm{p}=0.026$ for shock; Table 2).

Among the 45 ARDS patients, 24 (53.3\%) were treated with high flow nasal cannula (mild: 8 [53.3\%], moderate: 10 [55.6\%], severe: 6 [50.0\%]). Forty-five (100\%) patients received mechanical ventilation (mild: 15 [100\%], moderate: 18 [100\%], severe: 12 [100\%]), including 40 (88.9\%) patients that were on non-invasive ventilation and $9(20 \%)$ patients that received invasive mechanical ventilation; $15(33.3 \%)$ patients required prone position ventilation (mild: 1 [6.7\%], moderate: 6 [33.3\%], severe: 9 [75.0\%]). Additionally, 45 (100\%) patients were treated with the antiviral agents arbidol (mild: 15 [100\%], moderate: 18 [100\%], severe: 12 [100\%]). Thirty-eight (84.4\%) patients received the glucocorticoid methylprednisolone (mild: 12 [80\%], moderate: 15 [83.3\%], severe: 11 [91.7\%]), and $32(71.1 \%)$ received immunoglobulin (mild: 7 [46.7\%], moderate: 15 [83.3\%], severe: 10 [83.3\%]). Interferon (thymalfasin), the anti-bacterial agent (lopinavir), and chloroquine were administered to 8 (17.8\%), 6 (13.3\%), and 4 
Table 2. Comorbidities and prognosis of COVID-19 patients who developed ARDS.

\begin{tabular}{|c|c|c|c|c|c|}
\hline & $\begin{array}{l}\text { All patients } \\
(\mathrm{n}=45)\end{array}$ & $\begin{array}{c}\text { Mild } \\
(\mathrm{n}=15)\end{array}$ & $\begin{array}{l}\text { Moderate } \\
(\mathrm{n}=18)\end{array}$ & $\begin{array}{l}\text { Severe } \\
(\mathrm{n}=12)\end{array}$ & $p$ values \\
\hline Systolic pressure, median (IQR), mmHg & $123(112-152)$ & $116(112-137)$ & $124(113-147)$ & $115(88-125)$ & 0.469 \\
\hline Respiratory rate, median (IQR) & $30.5(30-35)$ & $30(29-31)$ & $34.5(30.3-36.5)$ & $33(29.3-39)$ & 0.039 \\
\hline PH, median (IQR) & $7.45(7.42-7.48)$ & $7.42(7.4-7.43)$ & $7.47(7.45-7.49)$ & $7.47(7.42-7.48)$ & 0.001 \\
\hline $\mathrm{PO}_{2}$, median (IQR) & $68(55.5-75)$ & $75(72-80)$ & $66.4(59.5-74.3)$ & $53.2(45.6-56.8)$ & $<0.001$ \\
\hline $\mathrm{PCO}_{2}$, median (IQR) & $35.6(31.8-41.1)$ & $40.2(32.8-41.4)$ & $34.3(31.6-37.2)$ & $35.5(30.4-41.2)$ & 0.363 \\
\hline $\mathrm{PO}_{2} / \mathrm{FiO}_{2}$, median (IQR) & $150(92.7-218)$ & $228(219.5-245)$ & $138.5(106.3-168.4)$ & $78.3(72.5-86.8)$ & $<0.001$ \\
\hline $\begin{array}{l}\text { Acute Physiology and Chronic Health } \\
\text { Evaluation II }\end{array}$ & $16(12-24)$ & $12(10-16)$ & $16(12-20)$ & $28(23-37)$ & $<0.001$ \\
\hline Glasgow Coma Scale score & $14(9-15)$ & $15(14-15)$ & $12.5(11.25-15)$ & $8(7-15)$ & 0.025 \\
\hline Sequential Organ Failure Assessment scores & $4(3-6)$ & $3(2.5-4)$ & $4(3-5)$ & $7(5.75,9.25)$ & $<0.001$ \\
\hline \multicolumn{6}{|l|}{ Comorbidities } \\
\hline Acute kidney injury & $7(15.6 \%)$ & $2(13.3 \%)$ & $2(11.1 \%)$ & $3(25 \%)$ & 0.592 \\
\hline Cardiac injury & $9(20 \%)$ & $1(6.7 \%)$ & $2(11.1 \%)$ & $6(50.0 \%)$ & 0.013 \\
\hline Liver dysfunction & $20(44.4 \%)$ & $4(26.7 \%)$ & $11(61.1 \%)$ & $5(41.7 \%)$ & 0.138 \\
\hline Hyperglycaemia & $12(26.7 \%)$ & $1(6.7 \%)$ & $6(33.3 \%)$ & $5(41.7 \%)$ & 0.072 \\
\hline Pneumothorax & $2(4.4 \%)$ & $0(0 \%)$ & $0(0 \%)$ & $2(16.7 \%)$ & 0.067 \\
\hline Shock & $5(11.1 \%)$ & $0(0 \%)$ & $1(5.6 \%)$ & $4(33.4 \%)$ & 0.026 \\
\hline \multicolumn{6}{|l|}{ Treatment } \\
\hline High flow nasal cannula & $24(53.3 \%)$ & $8(53.3 \%)$ & $10(55.6 \%)$ & $6(50.0 \%)$ & $>0.99$ \\
\hline Mechanical ventilation & $45(100 \%)$ & $15(100 \%)$ & $18(100 \%)$ & $12(100 \%)$ & $>0.99$ \\
\hline Non-invasive & $40(88.9 \%)$ & $15(100 \%)$ & $16(88.9 \%)$ & $9(75.0 \%)$ & 0.133 \\
\hline Invasive & $9(20 \%)$ & $0(0 \%)$ & $3(16.7 \%)$ & $6(50 \%)$ & 0.005 \\
\hline Prone position ventilation & $15(33.3 \%)$ & $1(6.7 \%)$ & $6(33.3 \%)$ & $9(75.0 \%)$ & 0.001 \\
\hline Antiviral agents & $45(100 \%)$ & $15(100 \%)$ & $18(100 \%)$ & $12(100 \%)$ & $>0.99$ \\
\hline Antibacterial agents & $6(13.3 \%)$ & $0(0 \%)$ & $1(5.6 \%)$ & $5(41.7 \%)$ & 0.004 \\
\hline Glucocorticoids & $38(84.4 \%)$ & $12(80.0 \%)$ & $15(83.3 \%)$ & $11(91.7 \%)$ & 0.774 \\
\hline Inhibitor & $4(8.9 \%)$ & $2(13.3 \%)$ & $2(11.1 \%)$ & $0(0 \%)$ & 0.551 \\
\hline Interferon & $8(17.8 \%)$ & $0(0 \%)$ & $3(16.7 \%)$ & $5(41.7 \%)$ & 0.017 \\
\hline Immunoglobulin & $32(71.1 \%)$ & $7(46.7 \%)$ & $15(83.3 \%)$ & $10(83.3 \%)$ & 0.058 \\
\hline \multicolumn{6}{|l|}{ Prognosis } \\
\hline Hospitalisation & $22(48.9 \%)$ & $5(33.3 \%)$ & $11(61.1 \%)$ & $6(50 \%)$ & 0.289 \\
\hline Discharge & $16(35.6 \%)$ & $9(60.0 \%)$ & $6(33.3 \%)$ & $1(8.3 \%)$ & 0.02 \\
\hline Death & $7(15.6 \%)$ & $1(6.7 \%)$ & $1(5.6 \%)$ & $5(41.7 \%)$ & 0.018 \\
\hline
\end{tabular}

Data are median (IQR), $\mathrm{n}(\%)$, or $\mathrm{n} / \mathrm{N}(\%)$, where $\mathrm{N}$ is the total number of patients with available data. P-values comparing mild, moderate, and severe groups were calculated using the $\chi^{2}$ test, Fisher's exact test, or Kruskal-Wallis $\mathrm{H}$ test. 
(8.9\%) patients, respectively (Table 2). Compared with patients with mild ARDS and moderate ARDS, patients with severe ARDS were more likely to require invasive mechanical ventilation $(p=0.005)$, prone position ventilation $(p=0.001)$, antibacterial agents $(\mathrm{p}=0.004)$, and interferon $(\mathrm{p}=0.017)$. Although antiviral agents $(n=12 ; 100 \%)$ and glucocorticoids $(n=11 ; 91.7 \%)$ were widely used in patients with severe ARDS, there was no significant difference in the frequency of the use of these agents among the different groups (Table 2).

\subsection{Survival Curves for Patients with Different ARDS Severity}

Among the 45 patients with ARDS, 22 (48.9\%) patients remained hospitalized (mild: 5 [33.3\%], moderate: 11 [61.1\%], severe: 6 [50\%]), 16 (35.6\%) were discharged (mild: 9 [60.0\%], moderate: 6 [33.3\%], severe: 1 [8.3\%]), and 7 (15.6\%) died (mild: 1 [6.7\%], moderate: 1 [5.6\%], severe: 5 [41.7\%]). Compared with patients with mild and moderate ARDS, patients with severe ARDS had a higher mortality rate $(\mathrm{p}=0.018$; Table 2$)$. Among discharged patients $(\mathrm{n}=16)$, the median hospital stay was 29 days (IQR, 19.0 - 38.0). Among patients that died of ARDS ( $\mathrm{n}=7$ ), the median duration from admission to death was 14 (IQR, 7 17) days. Kaplan Meier survival analysis showed significant differences between patients with different ARDS severity (log-rank tests, $p=0.034$ ) (Figure 1).

\subsection{Laboratory Findings}

Forty-five patients with ARDS had a red blood cell count of $3.81 \times 10^{9} / \mathrm{L}$ (IQR, 3.32 - 4.02; mild: $5.78 \times 10^{9} / \mathrm{L}$ [IQR, $3.9-7.2$ ], moderate: $8.2 \times 10^{9} / \mathrm{L}$ [IQR, 4.8 11.7], severe: $8.99 \times 10^{9} / \mathrm{L}$ [IQR, $7.25-13.22$ ]; $\mathrm{p}=0.564$ ), hemoglobin level of 118 g/L (IQR, 99 - 127; mild: 112.5 [IQR, 96.9 - 125.8], moderate: 116 [IQR, 105 - 129], severe: 102 [IQR, 87.7 - 120]; $\mathrm{p}=0.224)$, platelet count of $155 \mathrm{~g} / \mathrm{L}$ (IQR, 114 - 197; mild: 143 [IQR, 122 - 182.5], moderate: 149.5 [IQR, 101.2 - 194.5], severe: 177.5 [IQR, 140 - 215.7]; $\mathrm{p}=0.62$ ), activated partial thromboplastin time of $27.2 \mathrm{~s}$ (IQR, 22.6 - 32.4; mild: $27.2 \mathrm{~s}$ [IQR, 22.7 - 30.15], moderate: $27.15 \mathrm{~s}$ [IQR,

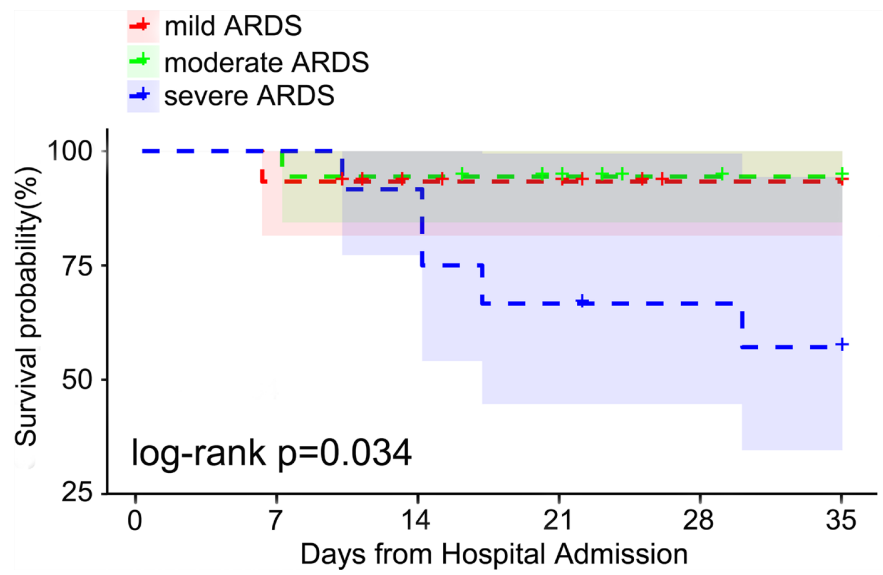

Figure 1. Survival of COVID-19 patients who developed ARDS. Red, green, and blue regions represent $95 \%$ CIs for patients with mild ARDS, moderate ARDS, and severe ARDS, respectively. 
22.02 - 33.52], severe: $30.8 \mathrm{~s}$ [IQR, 24.75 - 34.1]; $\mathrm{p}=0.704)$, fibrinogen level of 3.53 g/L (IQR, 2.76 - 4.65; mild: 3.69 [IQR, 3.2 - 4.1], moderate: 3.65 [IQR, 2.64 4.61], severe: 3.13 [IQR, 2.58 - 6.47]; $\mathrm{p}=0.981$ ), alanine aminotransferase level of 35 U/L (IQR, 18 - 64; mild: 32 [IQR, 18 - 42.5], moderate: 53 [IQR, 29.25 89.5], severe: 32 [IQR, 25.7 - 65.7]; $\mathrm{p}=0.296$ ), aspartate aminotransferase level of 31 U/L (IQR, 24 - 49; mild: 27 [IQR, 24.5 - 36.5], moderate: 41 [IQR, 26.25 56.75], severe: 33 [IQR, 24.75 - 34.1]; $\mathrm{p}=0.155)$, potassium ion level of 4.3mmol/L (IQR, 3.9 - 4.7; mild: 4.3 [IQR, 3.95 - 4.65], moderate: 4.2 [IQR, 3.9 4.75]; severe: 4.35 [IQR, 3.9 - 4.7]; $\mathrm{p}=0.916$ ), creatine kinase level of 47U/L (IQR, 27.5 - 134.5; mild: 37 [IQR, 29.5 - 100.75], moderate: 63 [IQR, 21 - 148], severe: 46 [IQR, 33.75 - 99.0]; $\mathrm{p}=0.962$ ), creatine kinase-MB level of 16U/L (IQR, 10.5 - 20.5; mild: 11.5 [IQR, 9 - 18.75], moderate: 15 [IQR, 12 - 20], severe: 19 [IQR, 15.5 - 27.0]; p = 0.09), clutamy transpeptidase level of 52U/L (IQR, 24 87; mild: 43 [IQR, 22 - 53], moderate: 52 [IQR, 24 - 92.25], severe: 65 [IQR, 43.75 - 157.5]; $\mathrm{p}=0.087)$, creatinine level of $65.4 \mu \mathrm{mol} / \mathrm{L}(\mathrm{IQR}, 58.9$ - 79.7; mild: 75.8 [IQR, 57.4 - 85.7], moderate: 62.45 [IQR, 58.25 - 73.7], severe: 63.9 [IQR, 61.3 - 79.7]; $\mathrm{p}=0.746)$, high-sensitivity troponin, $8 \mathrm{pg} / \mathrm{mL}$ (IQR, 4.5 - 13.5; mild: 4.9 [IQR, 2.15 - 10.7], moderate: 8.25 [IQR, 4.65 - 12.52], severe: 14.7 [IQR, 5.15 - 34.97]; $\mathrm{p}=0.134$ ), and brain natriuretic peptide $52.7 \mathrm{pg} / \mathrm{mL}$ (IQR, 19.5 - 226.8; mild: 62.3 [IQR, 20.95 - 194.7], moderate: 30.25 [IQR, 16.05 - 137.5], severe: 122.5 [IQR, 34.15 - 466.95]; $\mathrm{p}=0.21$ ).

Compared with patients with mild and moderate ARDS, a higher portion of patients with severe ARDS had high white blood cell count (all patients: $7.1 \times$ $10^{9} / \mathrm{L}, \mathrm{IQR}, 5.5-10.9$; mild: $5.78 \times 10^{9} / \mathrm{L}$, IQR, $3.9-7.2$; moderate: $8.2 \times 10^{9} / \mathrm{L}$, IQR, 4.8 - 11.7; severe: $\left.8.99 \times 10^{9} / \mathrm{L}, \mathrm{IQR}, 7.25-13.22 ; \mathrm{p}=0.009\right)$, high neutrophil count (all patients: $6.3 \times 10^{9} / \mathrm{L}$, IQR, $4.08-10.1$; mild: $4.48 \times 10^{9} / \mathrm{L}$, IQR, 2.47 - 5.26; moderate: $7.02 \times 10^{9} / \mathrm{L}$, IQR, 3.57 - 10.2; severe: $8.24 \times 10^{9} / \mathrm{L}$, IQR, 6.73 - 12.6; $\mathrm{p}=0.001$ ), low lymphocyte count (all patients: $0.69 \times 10^{9} / \mathrm{L}, \mathrm{IQR}$, 0.42 - 1.1 ; mild: $1.1 \times 10^{9} / \mathrm{L}$, IQR, $0.71-1.34$; moderate: $0.73 \times 10^{9} / \mathrm{L}, \mathrm{IQR}, 0.6$ 0.9 ; severe: $0.26 \times 10^{9} / \mathrm{L}, \mathrm{IQR}, 0.21-0.36 ; \mathrm{p}<0.001$ ), low lymphocyte percentage (all patients: 9.7\%, IQR, 3.84\% - 17.9\%; mild: 18.5\%, IQR, 12.3\% - 29.35\%; moderate: $9.16 \%$, IQR, $7.55 \%$ - 15.65\%; severe: $2.95 \%$, IQR, $2.475 \%$ - 3.93\%; p < 0.001), low albumin levels (all patients: $35.8 \mathrm{~g} / \mathrm{L}, \mathrm{IQR}, 34.1$ - 38.2; mild: $37.4 \mathrm{~g} / \mathrm{L}$, IQR, 37.75 - 40.7; moderate: 36.6 g/L, IQR, 35.1 - 39.5; severe: 34 g/L, IQR, 31.9 34.4; $\mathrm{p}=0.004$ ), prolonged prothrombin time (all patients:11.3 s, IQR, 10.5 11.9; mild: 11.1 s, IQR, 10.75 - 11.7; moderate: 10.5 s, IQR, 9.77 - 11.67; severe: $11.8 \mathrm{~s}, \mathrm{IQR}, 11.45$ - 12.8; $\mathrm{p}=0.035$ ), increased D-dimer (all patients: $1.16 \mathrm{mg} / \mathrm{L}$, IQR, 0.51 - 3.28; mild: $0.46 \mathrm{mg} / \mathrm{L}$, IQR, 0.34 - 1.15; moderate: $1.15 \mathrm{mg} / \mathrm{L}$, IQR, 0.63 - 2.18; severe: $7 \mathrm{mg} / \mathrm{L}, \mathrm{IQR}, 4.4$ - 17.1; $\mathrm{p}<0.001$ ), high total bilirubin levels (all patients: $13 \mathrm{mmol} / \mathrm{L}, \mathrm{IQR}, 10.1$ - 21.8; mild: $10.7 \mathrm{mmol} / \mathrm{L}, \mathrm{IQR}, 7.7$ - 13.7; moderate: $13.5 \mathrm{mmol} / \mathrm{L}, \mathrm{IQR}, 11.3$ - 17.8; severe: $19.2 \mathrm{mmol} / \mathrm{L}, \mathrm{IQR}, 14.1$ - 23.6; $\mathrm{p}=0.021$ ), high lactate dehydrogenase levels (all patients: $252 \mathrm{U} / \mathrm{L}, \mathrm{IQR}, 208$ 331; mild: 213 U/L, IQR, 185 - 260; moderate: 260.5 U/L, IQR, 228.7 - 315.5; se- 
vere: $356 \mathrm{U} / \mathrm{L}, \mathrm{IQR}, 256.5$ - 466.7; $\mathrm{p}=0.009$ ), high blood urea nitrogen levels (all patients: $7.06 \mathrm{mmol} / \mathrm{L}, \mathrm{IQR}, 5.04$ - 9.8; mild: $5.04 \mathrm{mmol} / \mathrm{L}, \mathrm{IQR}, 3.42$ - 5.9; moderate: $6.98 \mathrm{mmol} / \mathrm{L}, \mathrm{IQR}, 5.37$ - 9.57; severe: $9.4 \mathrm{mmol} / \mathrm{L}, \mathrm{IQR}, 7.5$ - 11.9; $\mathrm{p}=$ 0.003), and high C-reactive protein levels (all patients: $13.6 \mathrm{mg} / \mathrm{L}, \mathrm{IQR}, 3.97$ 42.01; mild: $7.02 \mathrm{mg} / \mathrm{L}, \mathrm{IQR}, 2.27$ - 14.9; moderate: $13.19 \mathrm{mg} / \mathrm{L}, \mathrm{IQR}, 4.39$ 16.44; severe: $60 \mathrm{mg} / \mathrm{L}, \mathrm{IQR}, 14.5$ - 121; $\mathrm{p}=0.013$; Table 3).

Table 3. Laboratory findings in COVID-19 patients who developed ARDS.

\begin{tabular}{|c|c|c|c|c|c|c|}
\hline & $\begin{array}{l}\text { Normal } \\
\text { range }\end{array}$ & $\begin{array}{l}\text { All patients } \\
(\mathrm{n}=45)\end{array}$ & $\begin{array}{c}\text { Mild } \\
(n=15)\end{array}$ & $\begin{array}{l}\text { Moderate } \\
(n=18)\end{array}$ & $\begin{array}{l}\text { Severe } \\
(n=12)\end{array}$ & $\begin{array}{c}\mathrm{p} \\
\text { values }\end{array}$ \\
\hline $\begin{array}{l}\text { White blood cell count, } \times 10^{9} \text { per } \\
\mathrm{L}\end{array}$ & $3.5-9.5$ & $7.1(5.5-10.9)$ & $5.78(3.9-7.2)$ & $8.2(4.8-11.7)$ & $8.99(7.25-13.22)$ & 0.009 \\
\hline Neutrophil count, $\times 10^{9}$ per $\mathrm{L}$ & $1.8-6.3$ & $6.3(4.08-10.1)$ & $4.48(2.47-5.26)$ & $7.02(3.57-10.2)$ & $8.24(6.73-12.6)$ & 0.001 \\
\hline Lymphocyte count, $\times 10^{9}$ per $\mathrm{L}$ & $1.1-3.2$ & $0.69(0.42-1.1)$ & $1.1(0.71-1.34)$ & $0.73(0.6-0.9)$ & $0.26(0.21-0.36)$ & $<0.001$ \\
\hline Lymphocyte percentage, $\%$ & $20-50$ & $9.7(3.84-17.9)$ & $18.5(12.3-29.35)$ & $9.16(7.55-15.65)$ & $2.95(2.475-3.93)$ & $<0.001$ \\
\hline Red blood cell count, $\times 10^{9}$ per $\mathrm{L}$ & $3.8-5.1$ & $3.81(3.32-4.02)$ & $3.75(3.16-4.02)$ & $3.7(3.27-4.07)$ & $3.91(3.61-4.12)$ & 0.564 \\
\hline Haemoglobin, $g / L$ & $115-150$ & $118(99-127)$ & $112.5(96.9-125.8)$ & $116(105-129)$ & $102(87.7-120)$ & 0.224 \\
\hline Platelet count, $\times 10^{9}$ per $\mathrm{L}$ & $125-350$ & $155(114-197)$ & $143(122-182.5)$ & $149.5(101.2-194.5)$ & $177.5(140-215.7)$ & 0.62 \\
\hline Prothrombin time, s & $9.9-12.5$ & $11.3(10.5-11.9)$ & $11.1(10.75-11.7)$ & $10.5(9.77-11.67)$ & $11.8(11.45-12.8)$ & 0.035 \\
\hline $\begin{array}{l}\text { Activated partial } \\
\text { thromboplastin time, s }\end{array}$ & $23-38$ & $27.2(22.6-32.4)$ & $27.2(22.7-30.15)$ & $27.15(22.02-33.52)$ & $30.8(24.75-34.1)$ & 0.704 \\
\hline D-dimer, mg/L & $\leq 0.50$ & $1.16(0.51-3.28)$ & $0.46(0.34-1.15)$ & $1.15(0.63-2.18)$ & $7(4.4-17.1)$ & $<0.001$ \\
\hline Fibrinogen, g/L & $1.8-3.5$ & $3.53(2.76-4.65)$ & $3.69(3.2-4.1)$ & $3.65(2.64-4.61)$ & $3.13(2.58-6.47)$ & 0.981 \\
\hline Total bilirubin, $\mathrm{mmol} / \mathrm{L}$ & $2-20.4$ & $13(10.1-21.8)$ & $10.7(7.7-13.7)$ & $13.5(11.3-17.8)$ & $19.2(14.1-23.6)$ & 0.021 \\
\hline Albumin, g/L & $35-55$ & $35.8(34.1-38.2)$ & $37.4(37.75-40.7)$ & $36.6(35.1-39.5)$ & $34(31.9-34.4)$ & 0.004 \\
\hline Alanine aminotransferase, $\mathrm{U} / \mathrm{L}$ & $1-40$ & $35(18-64)$ & $32(18-42.5)$ & $53(29.25-89.5)$ & $32(25.7-65.7)$ & 0.296 \\
\hline Aspartate aminotransferase, U/L & $2-42$ & $31(24-49)$ & $27(24.5-36.5)$ & $41(26.25-56.75)$ & $33(24.75-34.1)$ & 0.155 \\
\hline Potassium ion, $\mathrm{mmol} / \mathrm{L}$ & $3.5-5.5$ & $4.3(3.9-4.7)$ & $4.3(3.95-4.65)$ & $4.2(3.9-4.75)$ & $4.35(3.9-4.7)$ & 0.916 \\
\hline Creatine Kinase, U/L & $26-140$ & $47(27.5-134.5)$ & $37(29.5-100.75)$ & $63(21-148)$ & $46(33.75-99.0)$ & 0.962 \\
\hline Creatine Kinase-MB, U/L & $\leq 20$ & $16(10.5-20.5)$ & $11.5(9-18.75)$ & $15(12-20)$ & $19(15.5-27)$ & 0.09 \\
\hline Glutamyl transpeptidase, U/L & $\leq 50$ & $52(24-87)$ & $43(22-53)$ & $52(24-92.25)$ & $65(43.75-157.5)$ & 0.087 \\
\hline Blood urea nitrogen, $\mathrm{mmol} / \mathrm{L}$ & $1.8-7.00$ & $7.06(5.04-9.8)$ & $5.04(3.42-5.9)$ & $6.98(5.37-9.57)$ & $9.4(7.5-11.9)$ & 0.003 \\
\hline Creatinine, $\mu \mathrm{mol} / \mathrm{L}$ & $44-106$ & $65.4(58.9-79.7)$ & $75.8(57.4-85.7)$ & $62.45(58.25-73.7)$ & $63.9(61.3-79.7)$ & 0.746 \\
\hline Lactate dehydrogenase, U/L & $100-240$ & $252(208-331)$ & $213(185-260)$ & $260.5(228.7-315.5)$ & $356(265.5-466.7)$ & 0.009 \\
\hline High-sensitivity troponin, $\mathrm{pg} / \mathrm{mL}$ & $\leq 34.2$ & $8(4.5-13.5)$ & $4.9(2.15-10.7)$ & $8.25(4.65-12.52)$ & $14.7(5.17-34.97)$ & 0.134 \\
\hline Brain natriuretic peptide, $\mathrm{pg} / \mathrm{mL}$ & $\leq 100$ & $52.7(19.5-226.8)$ & $62.3(20.95-194.7)$ & $30.25(16.05-137.5)$ & $122.5(34.15-466.95)$ & 0.21 \\
\hline $\mathrm{C}$-reactive protein, $\mathrm{mg} / \mathrm{L}$ & $\leq 8$ & $13.6(3.97-42.01)$ & $7.02(2.27-14.9)$ & $13.19(4.39-16.44)$ & $60(14.5-121)$ & 0.013 \\
\hline
\end{tabular}

Data are median (IQR), $\mathrm{n}(\%)$, or $\mathrm{n} / \mathrm{N}(\%)$, where $\mathrm{N}$ is the total number of patients with available data. P-values comparing mild, moderate, and severe groups were calculated using the $\chi^{2}$ test, Fisher's exact test, or Kruskal-Wallis $\mathrm{H}$ test. 


\subsection{Multivariate Cox Regression Analysis of Prognostic Factors}

Multivariate Cox proportional models were used to identify prognostic factors in ARDS; HR values are shown in Table 4. Factors associated with mild ARDS included sex male (HR, 2.50; 95\% CI, $0.60-10.34, \mathrm{p}=0.206$ ), age > 65 (HR, 0.67; 95\% CI, $0.17-2.67, \mathrm{p}=0.566)$, diabetes $(\mathrm{HR}, 2.62 ; 95 \% \mathrm{CI}, 0.21-10.34, \mathrm{p}=$ 0.452), hypertension (HR, 1.05; 95\% CI, $0.26-4.26, \mathrm{p}=0.984$ ), white blood cell count (HR, 5.33; 95\% CI, $0.89-32.16, \mathrm{p}=0.068$ ), neutrophil count (HR, 5.71; 95\% CI, 1.15 - 28.35, p = 0.033), lymphocyte count (HR, 0.57; 95\% CI, 0.05 -

Table 4. Multivariate Cox regression of factors associated with ARDS development in COVID-19 patients.

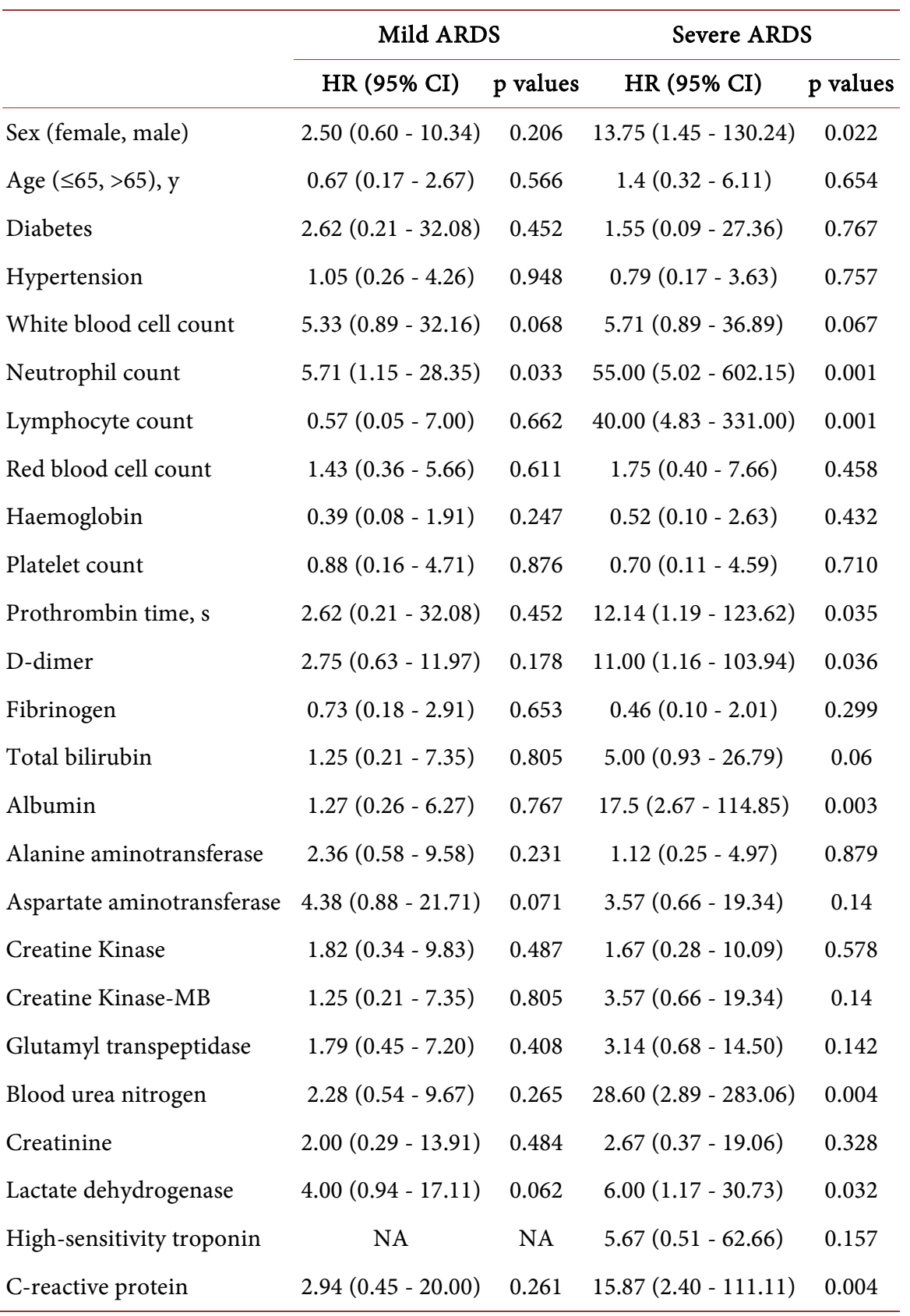


7.00, $\mathrm{p}=0.662)$, red blood cell count (HR, 1.43; 95\% CI, $0.36-5.66, \mathrm{p}=0.611$ ), hemoglobin levels ( $\mathrm{HR}, 0.39 ; 95 \% \mathrm{CI}, 0.08-1.91, \mathrm{p}=0.247)$, platelet count $(\mathrm{HR}$, 0.88 ; 95\% CI, $0.16-4.71, \mathrm{p}=0.876)$, prothrombin time (HR, 2.62; 95\% CI, 0.21 32.08, $\mathrm{p}=0.452)$, D-dimer $(\mathrm{HR}, 2.75$; 95\% CI, 0.63 - 11.97, $\mathrm{p}=0.178)$, fibrinogen levels (HR, 0.73; 95\% CI, 0.18 - 2.91, $\mathrm{p}=0.653)$, total bilirubin levels $(\mathrm{HR}, 1.25$; $95 \% \mathrm{CI}, 0.21-7.35, \mathrm{p}=0.805$ ), albumin levels (HR, 1.27; 95\% CI, $0.26-6.27, \mathrm{p}=$ 0.767), alanine aminotransferase levels ( $\mathrm{HR}, 2.36$; 95\% CI, $0.58-9.58, \mathrm{p}=0.231$ ), aspartate aminotransferase levels ( $\mathrm{HR}, 4.38 ; 95 \% \mathrm{CI}, 0.88-21.71, \mathrm{p}=0.071)$, creatine kinase levels (HR, 1.82; 95\% CI, 0.34 - 9.83, p = 0.487), creatine kinase-MB levels ( $\mathrm{HR}, 1.25$; 95\% CI, $0.21-7.35, \mathrm{p}=0.805)$, glutamyl transpeptidase (HR, 1.79; 95\% CI, $0.45-7.20, \mathrm{p}=0.408)$, blood urea nitrogen $(\mathrm{HR}, 2.28$; 95\% CI, 0.54 - 9.67, $\mathrm{p}=0.265)$, creatinine levels (HR, 2.00; 95\% CI, 0.29 - 13.91, $\mathrm{p}=0.484)$, lactate dehydrogenase levels (HR, 4.00; 95\% CI, $0.94-17.11, \mathrm{p}=$ 0.062), C-reactive protein levels ( $\mathrm{HR}, 2.94 ; 95 \% \mathrm{CI}, 0.45-20.00, \mathrm{p}=0.261$ ). These results suggest that neutrophil count is a significant risk factor associated with mild ARDS.

Factors associated with severe ARDS included age $>65$ (HR, 1.4; 95\% CI, 0.32 - 6.11, p = 0.654), diabetes (HR, 1.55; 95\% CI, 0.09 - 27.36, $\mathrm{p}=0.767)$, hypertension (HR, 0.79; 95\% CI, 0.17 - 3.63, $\mathrm{p}=0.757)$, red blood cell count $(\mathrm{HR}, 1.75$; 95\% CI, 0.40 - 7.66, p = 0.458), hemoglobin levels (HR, 0.52; 95\% CI, 0.10 - 2.63, $\mathrm{p}=0.432)$, platelet count $(\mathrm{HR}, 0.70 ; 95 \% \mathrm{CI}, 0.11-4.59, \mathrm{p}=0.710)$, fibrinogen levels (HR, 0.46; 95\% CI, 0.10 - 2.01, p = 0.299), alanine aminotransferase levels (HR, 1.12; 95\% CI, $0.25-4.97, \mathrm{p}=0.879)$, aspartate aminotransferase levels (HR, 3.57; 95\% CI, 0.66 - 19.34, $\mathrm{p}=0.14)$, creatine kinase levels ( $\mathrm{HR}, 1.67$; 95\% CI, 0.28 - 10.09, $\mathrm{p}=0.578$ ), creatine kinase-MB levels (HR, 3.57; 95\% CI, 0.66 19.34, $\mathrm{p}=0.14$ ), glutamyl transpeptidase levels (HR, 3.14; 95\% CI, $0.68-14.50, \mathrm{p}$ $=0.142)$, creatinine levels $(\mathrm{HR}, 2.67 ; 95 \% \mathrm{CI}, 0.37-19.06, \mathrm{p}=0.328)$, and high-sensitivity troponin levels $(\mathrm{HR}, 5.67 ; 95 \% \mathrm{CI}, 0.51-62.66, \mathrm{p}=0.157)$. Factors related to the development of severe ARDS included male sex (HR, 13.75; 95\% CI, 1.45 - 130.24, p = 0.022), neutrophil count (HR, 55.00; 95\% CI, 5.02 602.15, $\mathrm{p}=0.001)$, lymphocyte count (HR, 40.00; 95\% CI, $4.83-331.00, \mathrm{p}=$ $0.001)$, prothrombin time (HR, 12.14; 95\% CI, $1.19-123.62, \mathrm{p}=0.035)$, D-dimer levels (HR, 11.00; 95\% CI, 1.16 - 103.94, $\mathrm{p}=0.036)$, total bilirubin levels (HR, 5.00; 95\% CI, 0.93 - 26.79, p = 0.06), albumin levels (HR, 17.5; 95\% CI, 2.67 $114.85, \mathrm{p}=0.003)$, blood urea nitrogen $(\mathrm{HR}, 28.60 ; 95 \% \mathrm{CI}, 2.89-283.06, \mathrm{p}=$ $0.004)$, lactate dehydrogenase ( $\mathrm{HR}, 6.00 ; 95 \% \mathrm{CI}, 1.17-30.73, \mathrm{p}=0.032)$, and C-reactive protein levels (HR, 15.87; 95\% CI, 2.40 - 111.11, $\mathrm{p}=0.004)$ (Table 4).

\section{Discussion}

It has become evident that patients with COVID-19 are at risk of developing ARDS [4] [5] [6]. Severe ARDS is associated with increased mortality [9]. However, there is a lack of prognostic factors in COVID-19-associated severe ARDS. In this single-centered, retrospective, observational study, we stratified patients 
into three groups (mild, moderate, and severe ARDS) according to Berlin severity definitions. We assessed differences in clinical characteristics and outcomes among the different patient groups and found that men were more likely to develop severe ARDS. We also found that patients with severe ARDS were more likely to experience severe respiratory failure, sepsis, cardiac injury, and shock. Laboratory findings, including high neutrophil counts, low lymphocyte count, low albumin levels, prolonged prothrombin time, and high levels of D-dimer, lactate dehydrogenase, blood urea nitrogen, and C-reactive protein, were significantly associated with the risk of severe ARDS development. Laboratory indicators, such as neutrophil count and lymphocyte counts, could play an important role in the diagnosis of severe ARDS and guide clinical decision-making for patients with ARDS.

Several studies reported that nearly $70 \%$ of patients confirmed with COVID-19 were men [1] [2]. Moreover, elderly patients have a higher COVID-19-associated mortality rate than younger individuals [4]. These findings suggest that older men are the most susceptible to COVID-19 infection [2]. Consistently, we found that of the 45 enrolled ARDS patients, a high proportion included elderly or male patients. Furthermore, we found that males were more likely to develop severe ARDS than females $(p=0.044)$. However, the mechanism underlying this phenomenon remains unclear.

$\mathrm{Wu} \mathrm{C}$ et al. found that several factors associated with the development of ARDS, including fever, comorbidities, AST, creatinine, and glucose levels, were not associated with mortality [12]. In this study, we could not find an association between ARDS severity and disease signs and symptoms, including fever, dry cough, vomiting, fatigue, chest pain, hemoptysis, dorsalgia, and diarrhea). The presence of chronic diseases, including diabetes, hypertension, cardiovascular disease, chronic obstructive pulmonary disease, and cancer, were also not linked to the risk of severe ARDS development. Additionally, we found no association between ARDS severity and organ dysfunction, including acute kidney injury, liver dysfunction, and hyperglycemia.

In this study, we found that the incidence of severe ARDS was higher in patients with low lymphocyte count, elevated white blood cell count, and high neutrophil count. Neutrophilia has been observed in the peripheral blood and lungs of patients with COVID-19, and neutrophils were identified as the primary source of chemokines and cytokines [13] [14] [15] [16]. Patients with severe ARDS had a significantly higher neutrophil count compared with patients with mild or moderate ARDS. Elevated neutrophil numbers were likely due to host immune responses against the virus, contributing to cytokine storm. Lymphopenia has also been reported in patients with COVID-19 and has been suggested as a critical factor associated with disease severity [2] [5] [16] [17]. Consistent with previous reports that lymphopenia could increase inflammation [18], we found that compared to patients with mild or moderate ARDS, patients with severe ARDS had higher levels of C-reactive protein, a marker of generalized inflammation. 
Low albumin levels, prolonged prothrombin time, and high D-dimer, lactate dehydrogenase, total bilirubin, and blood urea nitrogen levels were associated with increased mortality in ARDS patients [4]. The predictive value of albumin and lactate dehydrogenase levels may be higher in monitoring the severity and course of ARDS in critically ill patients [19]. Previous studies demonstrated that prolonged prothrombin time and high $\mathrm{D}$-dimer were associated with excessive thrombin generation, inhibition of fibrinolysis, endothelial damage, and capillary leakage, which could increase the severity of ARDS [20]. Consistent with these results, we found that low albumin and prolonged prothrombin time, as well as high levels of D-dimer, total bilirubin, lactate dehydrogenase, and blood urea nitrogen, were significantly associated with the risk of severe ARDS development. These results suggest that laboratory findings could provide a powerful tool guiding severe ARDS diagnosis.

In this study, we found that ARDS development was associated with the presence of various dysfunctions and complications, such as severe respiratory failure, hypoxemia, sepsis, multiple systemic organ failure, and shock. We also found that the respiratory rate and $\mathrm{pH}$ were significantly higher in patients with severe ARDS, while the median $\mathrm{PO}_{2}$ and $\mathrm{PO}_{2} / \mathrm{FiO}_{2}$ were lower. The incidence of dyspnea was significantly higher in patients with severe ARDS, who required mechanical ventilation more frequently. Additionally, cardiac injury and shock were more prevalent among patients with severe ARDS. As patients with ARDS often develop severe hypoxemia and hypercapnia and most die of sepsis or multiorgan failure rather than refractory respiratory failure [7] [8], future studies are needed to explore the prognostic value of SOFA scores in patients with COVID-19.

This study has several limitations. Importantly, this was a single-center study with a limited sample size. Potential selection bias may have been introduced when identifying factors that influence clinical outcomes, and large multi-center studies are required to further define the clinical characteristics and risk factors of ARDS development in COVID-19 patients. Furthermore, this was a retrospective study; hence, the data of this study permit a preliminary assessment of the clinical course and outcomes of ARDS patients with COVID-19, and further studies are required to confirm our findings.

In conclusion, the mortality of COVID-19 patients with severe ARDS is considerably higher than that of patients with mild ARDS symptoms. Men with low lymphocyte count, high neutrophil count, prolonged prothrombin time, low levels of albumin, and high levels of D-dimer, lactate dehydrogenase, total bilirubin, blood urea nitrogen, and C-reactive protein have a higher risk of developing severe ARDS. Laboratory tests could play an important role in the diagnosis of severe ARDS and guide treatment decision-making for ARDS patients.

\section{Funding}

Supported by the Jingzhou science and technology development plan (key projects, 2015AC45 and 2016AE51-2), Hubei Province health and family 
planning scientific research project (key projects, WJ2017Z024, WJ2018H175, WJ2018H199, WJ2019M085 and WJ2019F126), and Hubei Provincial Natural Science Foundation of China (2018CFB775).

\section{Acknowledgements}

Cunjian Yi and Jinzhi Lu conceived and designed the study. They had full access to all data and were responsible for data analysis integrity and accuracy. Cunjian $\mathrm{Yi}$ and Jinzhi Lu wrote the manuscript. Xiaowen Wang, Liya Zhu, Xiangqiong Liu, Zhiqiang Liu, and Cunjian Yi revised the manuscript. Jian Liu, Keming Chen, Zhiqiang Liu, Chenqi Xin, and Yujie Chen performed the statistical analyses. All authors contributed to data acquisition, data analysis, or data interpretation and reviewed and approved the final version of the manuscript.

\section{Registration Number}

ChiCTR2000031836 (Chinese Clinical Trial Registry).

\section{Data Sharing}

The data that support the findings of this study are available from the corresponding author upon reasonable request. Participant data without names and identifiers will be made available after approval from the corresponding author and the National Health Commission. After the publication of study findings, the data will be available for others upon request. An email address will be provided for correspondence once data sharing is approved. A proposal with a detailed description of study objectives and statistical analysis will be required for the evaluation of data sharing requests by the corresponding author and the National Health Commission. Additional materials may also be needed during the process.

\section{Conflicts of Interest}

The authors declare no conflicts of interest regarding the publication of this paper.

\section{References}

[1] Chen, N., Zhou, M., Dong, X., et al. (2020) Epidemiological and Clinical Characteristics of 99 Cases of 2019 Novel Coronavirus Pneumonia in Wuhan, China: A Descriptive Study. The Lancet, 395, 507-513. https://doi.org/10.1016/S0140-6736(20)30211-7

[2] Huang, C., Wang, Y., Li, X., et al. (2020) Clinical Features of Patients Infected with 2019 Novel Coronavirus in Wuhan, China. The Lancet, 395, 497-506. https://doi.org/10.1016/S0140-6736(20)30183-5

[3] Holshue, M.L., DeBolt, C., Lindquist, S., et al. (2020) First Case of 2019 Novel Coronavirus in the United States. The New England Journal of Medicine. https://doi.org/10.1056/NEJMoa2001191

[4] Yang, X., Yu, Y., Xu, J., et al. (2020) Clinical Course and Outcomes of Critically Ill Patients with SARS-CoV-2 Pneumonia in Wuhan, China: A Single-Centered, Retrospective, Observational Study. The Lancet Respiratory Medicine, 8, 475-481. 
[5] Wang, D., Hu, B., Hu, C., et al. (2020) Clinical Characteristics of 138 Hospitalized Patients with 2019 Novel Coronavirus-Infected Pneumonia in Wuhan, China. JAMA, 323, 1061-1069. https://doi.org/10.1001/jama.2020.1585

[6] Albarello, F., Pianura, E., Di Stefano, F., et al. (2020) 2019-Novel Coronavirus Severe Adult Respiratory Distress Syndrome in Two Cases in Italy: An Uncommon Radiological Presentation. International Journal of Infectious Diseases, 93, 192-197. https://doi.org/10.1016/j.ijid.2020.02.043

[7] Abe, T., Madotto, F., Pham, T., et al. (2016) Epidemiology, Patterns of Care, and Mortality for Patients with Acute Respiratory Distress Syndrome in Intensive Care Units in 50 Countries. JAMA, 315, 788-800. https://doi.org/10.1001/jama.2016.0291

[8] Peck, T.J. and Hibbert, K.A. (2019) Recent Advances in the Understanding and Management of ARDS. F1000Research, 8, F1000.

https://doi.org/10.12688/f1000research.20411.1

[9] Force, A.D.T., Ranieri, V.M. and Rubenfeld, G.D. (2012) Acute Respiratory Distress Syndrome: The Berlin Definition. JAMA, 307, 2526-2533.

https://doi.org/10.1001/jama.2012.5669

[10] WHO (2020) Clinical Management of Severe Acute Respiratory Infection When Novel Coronavirus (nCoV) Infection Is Suspected.

https://www.who.int/publications/i/item/10665-332299

[11] Kidney Disease: Improving Global Outcomes (KDIGO) Acute Kidney Injury Work Group. KDIGO Clinical Practice Guideline for Acute Kidney Injury. March 2012. https://kdigo.org/wp-content/uploads/2016/10/KDIGO-2012-AKI-Guideline-Englis h.pdf

[12] Wu, C., Chen, X., Cai, Y., et al. (2020) Risk Factors Associated with Acute Respiratory Distress Syndrome and Death in Patients with Coronavirus Disease 2019 Pneumonia in Wuhan, China. JAMA Internal Medicine, 180, 934-943. https://doi.org/10.1001/jamainternmed.2020.0994

[13] Channappanavar, R. and Perlman, S. (2017) Pathogenic Human Coronavirus Infections: Causes and Consequences of Cytokine Storm and Immunopathology. Seminars in Immunopathology, 39, 529-539. https://doi.org/10.1007/s00281-017-0629-x

[14] Wang, Y.H., Lin, A.S. and Chao, T.Y. (2004) A Cluster of Patients with Severe Acute Respiratory Syndrome in a Chest Ward in Southern Taiwan. Intensive Care Medicine, 30, 1228-1231. https://doi.org/10.1007/s00134-004-2311-8

[15] Nicholls, J.M., Poon, L.L. and Lee, K.C. (2003) Lung Pathology of Fatal Severe Acute Respiratory Syndrome. The Lancet, 361, 1773-1778. https://doi.org/10.1016/S0140-6736(03)13413-7

[16] Xu, Z., Shi, L. and Wang, Y. (2020) Pathological Findings of COVID-19 Associated with Acute Respiratory Distress Syndrome. The Lancet Respiratory Medicine, 8, 420-422. https://doi.org/10.1016/S2213-2600(20)30076-X

[17] Chan, J.F., Yuan, S. and Kok, K.H. (2020) A Familial Cluster of Pneumonia Associated with the 2019 Novel Coronavirus Indicating Person-to-Person Transmission: A Study of a Family Cluster. The Lancet, 395, 514-523. https://doi.org/10.1016/S0140-6736(20)30154-9

[18] Zidar, D.A., Al-Kindi, S.G. and Liu, Y. (2019) Association of Lymphopenia with Risk of Mortality among Adults in the US General Population. JAMA Network Open, 2, e1916526. https://doi.org/10.1001/jamanetworkopen.2019.16526

[19] Hoeboer, S.H., Oudemans-van Straaten, H.M. and Groeneveld, A.B. (2015) Albumin Rather than C-Reactive Protein May Be Valuable in Predicting and Monitoring the Severity and Course of Acute Respiratory Distress Syndrome in Critically Ill Pa- 
tients with or at Risk for the Syndrome after New Onset Fever. BMC Pulmonary Medicine, 15, Article No. 22. https://doi.org/10.1186/s12890-015-0015-1

[20] Koyama, K., Katayama, S. and Tonai, K. (2019) Biomarker Profiles of Coagulopathy and Alveolar Epithelial Injury in Acute Respiratory Distress Syndrome with Idiopathic/Immune-Related Disease or Common Direct Risk Factors. Critical Care, 23, 283. https://doi.org/10.1186/s13054-019-2559-6 